\title{
Do Canadians Support Tailored and Targeted Public Health Programs? A Discrete Choice Experiment
}

Kiffer G. Card ( $\sim$ kcard@sfu.ca )

Simon Fraser University

Marina Adshade

University of British Columbia

Robert S. Hogg

Simon Fraser University

Jody Jollimore

Community Based Research Centre

Nathan J. Lachowsky

University of Victoria

\section{Research Article}

Keywords: Discrete Choice Experiment; Marginalized Communities, Prevention, Treatment, Infectious Disease, Mental Health, Substance Use, Chronic Disease, Resource Allocation Preferences

Posted Date: January 11th, 2022

DOI: https://doi.org/10.21203/rs.3.rs-1129112/v2

License: (c) (1) This work is licensed under a Creative Commons Attribution 4.0 International License. Read Full License 


\section{Abstract}

Background. We aimed to assess public support of tailored and targeted public health interventions for various marginalized communities.

Methods. We conducted a discrete choice experiment using a web-based survey advertised to Facebook and Instagram users living in Canada, aged $>16$. Participants were asked to choose between funding two hypothetical public health programs. Each program was described by its purpose; expected increase in life expectancy; and target group. Demographically-weighted generalized linear mixed-effects models were constructed to identify program factors associated with program selection.

Results. 23,889 exercises were completed by 3,054 participants. Selected programs were less likely to focus on prevention (vs. treatment). For each 1-year increase in the marginal years of life gained, there was a $15 \%$ increase in the odds of a program being selected. Interventions tailored to marginalized communities or targeting stigmatized health conditions were less likely to be selected compared with interventions targeted to the general population or targeting chronic health conditions. Noteworthy exceptions included an increased preference for interventions aligning with the perceived needs of marginalized communities (e.g. HIV and men who have sex with men).

Conclusions. Stigmatizing perceptions of health conditions and key populations likely influence public health programming preferences of Canadians. Informational campaigns highlighting disparities experienced by marginalized populations may improve support for targeted and tailored interventions.

\section{Introduction}

The rise of statistical thinking and evidence-based decision making has promoted the idea that public health decisions are shaped primarily by scientific findings. ${ }^{1,2}$ In reality, public health systems are socially influenced - meaning that while scientific findings may play an important role in decision making, the extent of their influence is mediated by the social environments within which they exist. ${ }^{3-6}$ In Canada, most public health activities are influenced by federal parliament, provincial legislative assemblies, ministerial bureaucracies, professional unions and colleges, healthcare organizations, and, of course, patients and providers themselves - not to mention interactions between these and the fundamental environmental and biological realities that put pressures on these systems. ${ }^{1,7}$ Public health is therefore vulnerable to and often serves to reproduce the biases of these individuals and institutions. ${ }^{8-10}$

Understanding how bias operates within these social environments is important for improving decisions regarding how policies are made, priorities are set, and resources are allocated. These decisions, after all, are the basis of public health programming: they determine what inputs and resources are mobilized, what activities are undertaken, and thereby shape program outputs and outcomes. In short, such decisions determine what harms are addressed, how, and for whom. ${ }^{11}$ The factors that enter into these decisions are diverse and manifold. They may include considerations of which conditions are most harmful or urgent to address, which are treatable or preventable, which populations are most greatly impacted, and the relative opportunity costs of addressing one problem over another. While these factors are undoubtedly worthy of consideration, the fact that they are socially constructed means they are vulnerable to bias. $^{12-14}$

It is increasingly recognized that biased policy decisions tend to disadvantage and harm marginalized communities. ${ }^{15}$ To prevent these harms, equity-oriented frameworks have been used to promote tailored and targeted public health interventions that aim to directly promote health and wellness among marginalized communities. ${ }^{16-19}$ Yet, the implementation of these frameworks and programs requires the support of policy-makers and the institutions that empower them. ${ }^{20}$ Thus, the very social conditions that have given rise to health disparities must be engaged in order to address them. This poses a considerable challenge to equity-oriented public health programs in many jurisdictions - particularly when marginalized communities continue to face stigma and discrimination.

Understanding how the public conceptualizes the need for tailored and targeted public health programs may help to address barriers to health equity. While the public's influence on health policy decisions is mediated through institutional processes, we nonetheless conceptualize public opinion as relatively important - especially when analyzing public health systems of developed democracies. This position is supported by previous work which suggests that public perceptions and support of health and social programs may play an important role in facilitating positive policy change. ${ }^{21}$ Previous research has examined the preferences of the public and policy makers, but these have usually focused on age, disease severity, and income, with little attention to specific marginalized identities/groups. ${ }^{22,22-26}$ To contribute to our understanding of public views regarding tailored and targeted public health programs for specific marginalized groups, we conducted a discrete choice experiment that elicited resource allocation preferences of adults in Canada. We hypothesized that individuals would favour programs designed for the general population, as opposed to those designed for marginalized communities or stigmatized health conditions. This hypothesis emerged from our anticipation that stigma likely influences how decisions about program selection are made.

\section{Methods}

\section{Participant Recruitment}

Participants for our study were recruited online using paid advertising on Facebook and Instagram between May 13th, 2020 and June 15th, 2020, during which time Canada was exiting the third wave of the COVID-19 pandemic and seeing breaking national headlines about mass burial sites at Indigenous residential schools ${ }^{27,28}$ (both of which are factors that we acknowledge may have considerable potential to influence perceptions on health spending). Recruitment proceeded for one month to support engagement of infrequent social media users. After clicking on a paid advertisement in either English or French, participants were screened for eligibility. Eligibility criteria restricted participation to individuals who (1) were 16 years of age or older, (2) reported living 
in Canada, (3) provided informed consent, and (4) were able to complete the questionnaire in English or French. Ethics review of our study protocol was conducted by the Research Ethics Board at the University of Victoria (\#H21-00244).

\section{Data Collection}

The survey questionnaire was hosted on the Qualtrics Platform. After being screened for eligibility and providing informed consent, participants participated in a discrete choice experiment (See Figure 1). The experiment consisted of 8 exercises in which participants were asked to imagine that they were a decision maker choosing which of two public health programs should be funded. Following previous studies utilizing discrete choice experiments to assess program preferences, each program was described by its (1) purpose, (2) expected increase in life expectancy, and its (3) target audience. JavaScript was used to randomly generate the descriptors for each program from a list of 12 program purposes (i.e., To prevent cancer; To prevent diabetes; To prevent drug overdoses; To prevent heart disease; To prevent mental health problems; To prevent sexually transmitted infections, including HIV; To treat cancer; To treat diabetes; To treat heart disease; To treat mental health problems; To treat sexually; transmitted infections, including HIV; To treat substance use and addiction), 10 life expectancy estimates (i.e., 1 -10 years), and 12 target populations (i.e., General Population; Gay, bisexual, and other men who have sex with men; People who use drugs; Indigenous people; African, Caribbean, and Black people; People engaged in sex work; Migrants and refugees; People living in or recently released from correctional facilities; Transgender people; People living with HIV; Youth and Young Adults; Seniors and Older Adults). Ethics review of our study protocol was conducted by the Research Ethics Board at the University of Victoria (\#H21-00244). All methods were carried out in accordance with relevant guidelines. All participants provided informed consent prior to participation in the study.

\section{Data Analysis}

To adjust for sampling biases, we conducted iterative proportional fitting using the anesrake package in $\mathrm{R}$ (v. 4.0.3). This allowed us to generate statistical weights that could be used in our descriptive and multivariable analyses. Target weights, reported in Table 1, were based off the Canadian Census Population (adjusting for age, gender, ethnicity, educational attainment, income, and province of residence). As the data were collected via an online, opt-in survey, we also adjusted for the average time participants spent using social media (based on estimates from the Ryerson Social Media Lab) ${ }^{29}$ and political orientation (based on estimates from CBC News Poll Tracker Averages) ${ }^{30}$. Descriptive statistics were used to provide an overall description of the sample and assess how frequently programs with each characteristic were selected when participants were presented with an opportunity to choose programs with each given characteristic. A weighted generalized linear mixed-effects model was constructed using the Ime4 package to identify program factors associated with program selection preferences. The outcome variable indicated whether the program was selected or not. A random effect term was used to account for within-person effects arising from the repeated observations (i.e., up to 8 per participant). Explanatory factors included measures of the program purpose, the target population, whether the intervention focused on a prevention or treatment approach, and the relative years of life gained for the selective (vs. unselected) program. An interaction term between the program purpose and target audience was included (12x12). 
Table 1

Participant Characteristics

\begin{tabular}{|c|c|c|c|}
\hline & Unweighted & Target & Weighted \\
\hline & $\mathrm{n}(\%)$ & $(\%)$ & $\mathrm{n}(\%)$ \\
\hline \multicolumn{4}{|l|}{ Age } \\
\hline 16 to 30 years & $165(5.4)$ & 22.6 & $552.8(18.1)$ \\
\hline 31 to 64 years & $1654(54.2)$ & 57.9 & $1823.1(59.7)$ \\
\hline 65 years or older & $1235(40.4)$ & 19.5 & $678.1(22.2)$ \\
\hline \multicolumn{4}{|l|}{ Gender } \\
\hline Man & $1227(40.2)$ & 49.3 & $1465.0(48.0)$ \\
\hline Woman & $1569(51.4)$ & 50.6 & $1584.7(51.9)$ \\
\hline Non-binary & $105(3.5)$ & 0.1 & $4.3(0.1)$ \\
\hline \multicolumn{4}{|l|}{ Province } \\
\hline Atlantic Canada & $264(8.6)$ & 6.6 & $220.7(7.2)$ \\
\hline British Columbia & $720(23.6)$ & 13.2 & $417.4(13.7)$ \\
\hline Ontario & 1018 (33.3) & 38.3 & $1200.5(39.3)$ \\
\hline The Prairies & $886(29.0)$ & 18.3 & $573.4(18.8)$ \\
\hline Quebec & $151(4.9)$ & 23.2 & $632.0(20.7)$ \\
\hline The Territories & $15(0.5)$ & 0.3 & $9.9(0.3)$ \\
\hline \multicolumn{4}{|l|}{ Ethnicity } \\
\hline African, Caribbean, or Black & $84(2.8)$ & 3.5 & $76.2(2.5)$ \\
\hline Indigenous & $159(5.2)$ & 4.9 & $190.0(6.2)$ \\
\hline White & $2237(73.2)$ & 72.8 & $2351.1(77.0)$ \\
\hline Other & $574(18.8)$ & 18.8 & $436.7(14.3)$ \\
\hline \multicolumn{4}{|l|}{ Educational Attainment } \\
\hline High School Diploma or Lower & $471(15.4)$ & 44.7 & $1335.0(43.8)$ \\
\hline Advanced Training, below Bachelors level & $1239(40.6)$ & 32.0 & $1008.4(33.1)$ \\
\hline Advanced Training, Bachelors level or Above & $1342(44.0)$ & 23.3 & $705.6(23.1)$ \\
\hline \multicolumn{4}{|l|}{ Income } \\
\hline Under $\$ 30,000$ & $578(18.9)$ & 45.1 & $1343.7(44.0)$ \\
\hline$\$ 30,000$ to less than $\$ 60,000$ & $779(25.5)$ & 21.2 & $722.0(23.6)$ \\
\hline$\$ 60,000$ to less than $\$ 90,000$ & $653(21.4)$ & 23.3 & $333.6(10.9)$ \\
\hline$\$ 90,000$ or more & 1044 (34.2) & 10.4 & $654.7(21.4)$ \\
\hline \multicolumn{4}{|l|}{ Political Affiliation, Federal } \\
\hline Bloc Québécois & $65(2.1)$ & 6.8 & $189.0(6.2)$ \\
\hline Conservative Party of Canada & $1432(46.9)$ & 28.8 & $967.8(31.7)$ \\
\hline Green Party of Canada & $294(9.6)$ & 4.6 & $234.6(7.7)$ \\
\hline Liberal Party of Canada & $563(18.4)$ & 35.3 & $1087.7(35.6)$ \\
\hline New Democratic Party (NDP) & $700(22.9)$ & 19.4 & $574.8(18.8)$ \\
\hline \multicolumn{4}{|l|}{ Time Spent on Social Media } \\
\hline Less than 30 minutes per day & $1677(54.9)$ & 50.0 & $1518.8(49.7)$ \\
\hline More than 30 minutes per day & $1377(45.0)$ & 50.0 & $1535.1(50.2)$ \\
\hline
\end{tabular}




\section{Results}

A total of 23,889 exercises were completed by 3,054 participants. Table 1 shows the unweighted and weighted descriptive statistics of the sample, as well as the target prevalence used in the creation of statistical weights. In brief, youth and young adults, men, residents of Quebec, less educated individuals, and lower income individuals were under represented in the survey, as were those who identified with the Liberal and Green federal political parties. Statistical weights were used to adjust for these biases, resulting in an analytic sample that closely represented the demographic characteristics of the Canadian population: $57.9 \%$ were between 31 and 64 years of age, $49.3 \%$ identified as male, $72.8 \%$ identified as white, $55.3 \%$ had received education or training beyond a high school diploma, and $54.9 \%$ had annual incomes greater than $\$ 30,000$ CAD.

Table 2 shows the proportion of time interventions were selected that focused on each health condition and target audience. Programs targeting the general population were the most frequently selected (70.5\%), followed by those supporting youth and young adults (68.3\%), Indigenous peoples (62.1\%), and seniors and older adults (57.8\%). Interventions targeting migrants and refugees (40.3\%), gay and bisexual men (36.1\%) and transgender people (33.4\%) were the least frequently selected. Figure 2 shows that as the number of life years gained for a given intervention increased, it was selected more frequently by participants. This was true for all sub-populations reviewed, though we did not test for differences in slopes. Interventions for mental health (56.0\%), cancer, (53.4\%), diabetes (53.2\%) and heart disease (51.6\%) were all selected at least half of the time, while those targeting substance use (47.7\%) and HIV or other sexually transmitted infections (43.5\%) were selected slightly less than half of the time they appeared.

Table 2

Proportion of time a program serving each target audience group was selected when displayed, stratified by health condition

\begin{tabular}{|c|c|c|c|c|c|c|c|}
\hline & $\begin{array}{l}\text { \% Population Selected, } \\
\text { Overall }\end{array}$ & $\begin{array}{l}\text { Mental } \\
\text { Health }\end{array}$ & Cancer & Diabetes & $\begin{array}{l}\text { Heart } \\
\text { Disease }\end{array}$ & $\begin{array}{l}\text { Substance } \\
\text { Use }\end{array}$ & $\begin{array}{l}\text { HIV \& } \\
\text { STIS }\end{array}$ \\
\hline \multirow[t]{2}{*}{$\%$ Condition Selected, Overall } & - & 56.0 & 53.4 & 53.2 & 51.6 & 47.7 & 43.5 \\
\hline & $\%$ & $\%$ & $\%$ & $\%$ & $\%$ & $\%$ & $\%$ \\
\hline General Population & 70.5 & 75.8 & 70.6 & 75.1 & 76.8 & 68.2 & 62.1 \\
\hline Youth and Young Adults & 68.3 & 77.2 & 70.8 & 71.9 & 65.7 & 67.3 & 61.9 \\
\hline Indigenous people & 62.1 & 66.0 & 69.2 & 68.2 & 62.4 & 60.0 & 54.1 \\
\hline Seniors and Older Adults & 57.8 & 68.5 & 60.6 & 67.4 & 64.5 & 54.6 & 40.4 \\
\hline African, Caribbean, and Black people & 49.6 & 51.8 & 59.7 & 57.1 & 56.8 & 42.4 & 40.2 \\
\hline People who use drugs & 47.0 & 56.8 & 46.7 & 44.9 & 42.9 & 51.2 & 40.0 \\
\hline People who are/have been incarcerated & 45.5 & 57.8 & 42.3 & 45.8 & 41.6 & 47.0 & 39.6 \\
\hline People living with HIV & 45.5 & 46.2 & 51.3 & 48.9 & 48.9 & 40.3 & 43.1 \\
\hline People engaged in sex work & 43.1 & 47.1 & 38.9 & 43.2 & 39.7 & 44.7 & 42.3 \\
\hline Migrants and refugees & 40.3 & 45.0 & 48.4 & 41.8 & 45.8 & 31.4 & 37.9 \\
\hline $\begin{array}{l}\text { Gay, bisexual, and other men who have sex with } \\
\text { men (gbMSM) }\end{array}$ & 36.1 & 38.7 & 40.7 & 35.7 & 38.9 & 33.9 & 33.0 \\
\hline Transgender people & 33.4 & 42.9 & 39.3 & 36.5 & 34.9 & 28.2 & 26.3 \\
\hline
\end{tabular}

Results of multivariable analyses are shown in Table 3. Selected programs were less likely to focus on prevention (vs. treatment; Adjusted Odds Ratios [aOR]= $0.87,95 \%$ Confidence Intervals $[\mathrm{Cl}]=0.84-0.91)$. For each 1 -year increase in the marginal years of life gained, there was a $15 \%$ increase in the odds of a program being selected $(\mathrm{aOR}=1.15,95 \% \mathrm{Cl}=1.14-1.15)$. Interventions targeting African, Caribbean, and $\mathrm{Black}$ people $(\mathrm{aOR}=0.56,95 \% \mathrm{Cl}=0.41-0.77) ;$ gay, bisexual, and other Men who have Sex with Men (gbMSM; aOR = 0.24, 95\% Cl=0.17-0.33)); migrants and refugees $(\mathrm{aOR}=0.26,95 \% \mathrm{Cl}=0.20-0.36)$; people engaged in sex work $(\mathrm{aOR}=0.24,95 \% \mathrm{Cl}=0.18-0.33)$; people who are/have been incarcerated $(\mathrm{aOR}=0.19,95 \% \mathrm{Cl}=0.14-0.26)$; people living with $\mathrm{HIV}(\mathrm{aOR}=$ $0.52,95 \% \mathrm{Cl}=0.37-0.72$ ); people who use drugs ( $\mathrm{aOR}=0.34,95 \% \mathrm{Cl}=0.25-0.47$ ); and transgender people $(\mathrm{aOR}=0.26,95 \% \mathrm{Cl}=0.19-0.36)$ were less likely to be selected compared with interventions targeted to the general population. Relative to interventions addressing cancer, those addressing diabetes (aOR = $1.56,95 \% \mathrm{Cl}=1.15-2.11)$ and heart disease $(\mathrm{aOR}=1.75,95 \% \mathrm{Cl}=1.30-2.34)$ were preferred, while those addressing HIV and other STI's had lower odds of being preferred $(\mathrm{aOR}=0.60,95 \% \mathrm{Cl}=0.46-0.78)$. The program purpose and target audience interaction term revealed notable exceptions to these patterns: $\mathrm{HIV}$ \& STI interventions for gbMSM $(\mathrm{aOR}=1.73,95 \% \mathrm{Cl}=1.18-2.53)$, migrants and refugees $(\mathrm{aOR}=1.91,95 \% \mathrm{Cl}=1.33-2.74)$, people engaged in sex work, $(\mathrm{aOR}=$ $1.78,95 \% \mathrm{Cl}=1.23-2.57)$, and people who are or have been incarcerated (aOR $=2.20,95 \% \mathrm{Cl}=1.53-3.16)$ were more likely to be selected, and $\mathrm{HIV} \mathrm{\&} \mathrm{STI}$ interventions targeting seniors and older adults were less likely to be selected $(\mathrm{aOR}=0.35,95 \% \mathrm{Cl}=0.24-0.50)$. Mental health interventions targeting Indigenous people $(\mathrm{aOR}=0.62,95 \% \mathrm{Cl}=0.42-0.93)$, people living with $\mathrm{HIV}(\mathrm{aOR}=0.61,95 \% \mathrm{Cl}=0.40-0.91)$, and seniors $/$ older adults $(\mathrm{aOR}=0.61,95 \% \mathrm{Cl}=$ 0.41-0.92) were less likely to be selected, while those targeting people who are or have been incarcerated $(\mathrm{aOR}=2.41,95 \% \mathrm{Cl}=1.64-3.56)$ or who used drugs $(\mathrm{aOR}=1.53,95 \% \mathrm{Cl}=1.03-2.28)$ were more likely to be selected. Similarly, substance use interventions targeted to people who are or have been incarcerated $(\mathrm{aOR}=1.92,95 \% \mathrm{Cl}=1.33-2.77)$ or people who used drugs $(\mathrm{aOR}=1.45,95 \% \mathrm{Cl}=1.00-2.11)$ were more likely to be selected, while those targeted to African, Caribbean, and Black people $(\mathrm{aOR}=0.60,95 \% \mathrm{Cl}=0.41-0.87$ ), people living with $\mathrm{HIV}(\mathrm{aOR}=0.58,95 \% \mathrm{Cl}=0.40-0.86)$, and seniors $/$ older adults $(\mathrm{aOR}=0.44$, $95 \% \mathrm{Cl}=0.30-0.65$ ) were less likely to be selected. Finally, interventions addressing diabetes or heart disease among all marginalized groups (except people who were or had been incarcerated) were less likely to be selected 
Table 3

Factors associated with whether a program was selected (vs. not selected), with Target Audience*Program Purpose Interaction

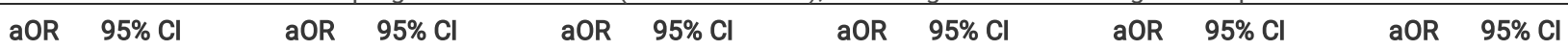

Treatment or
Prevention (R

Prevention (Ref:

Prevention

Difference in Years

of Life Gained ( $P e r$

1 year)

Client Population

(Ref: General

Population)

African, Caribbean, and Black people

gbMSM

Indigenous people

Migrants and

refugees

People engaged in

sex work

People who

are/have been

incarcerated

People living with

HIV

People who use

drugs

Seniors and Older

Adults

$\begin{array}{lcccc}\begin{array}{l}\text { Transgender } \\ \text { people }\end{array} & \mathbf{0 . 2 6} & \mathbf{0 . 1 9} & \mathbf{0 . 3 6} \quad- \\ \begin{array}{l}\text { Youth and Young } \\ \text { Adults }\end{array} & 1.22 & 0.88 & 1.70 \quad-\end{array}$

Health Condition

(Ref: Cancer)

Diabetes

$\begin{array}{lll}1.56 & 1.15 & 2.11\end{array}$

Heart disease

$\begin{array}{lll}1.75 & 1.30 & 2.34\end{array}$

HIV/STIS

Mental health

problems

Substance use

Target

Audience*Program

$\begin{array}{lll}0.60 & 0.46 & 0.78\end{array}$

Purpose

(Ref: General

Population)

African, Caribbean, $\quad 1.00$

0.4

Diabetes

Heart Disease

HIV/STIS

Mental Health

Substance Use

and Black people

Gay, bisexual, and $\quad 1.00$

other men who

have sex

with men

(gbMSM)

Indigenous people $\quad 1.00$

Migrants and

refugees

aOR = Adjusted Odds Ratio; $\mathrm{Cl}$ = Confidence Interval

\begin{tabular}{lllllllllllllll}
$\mathbf{0 . 5 5}$ & $\mathbf{0 . 3 6}$ & $\mathbf{0 . 8 2}$ & $\mathbf{0 . 3 2}$ & $\mathbf{0 . 2 1}$ & $\mathbf{0 . 4 7}$ & 0.83 & 0.58 & 1.19 & $\mathbf{0 . 6 2}$ & $\mathbf{0 . 4 2}$ & $\mathbf{0 . 9 3}$ & 0.75 & 0.52 & 1 \\
\hline $\mathbf{0 . 5 0}$ & $\mathbf{0 . 3 3}$ & $\mathbf{0 . 7 4}$ & $\mathbf{0 . 6 1}$ & $\mathbf{0 . 4 1}$ & $\mathbf{0 . 9 0}$ & $\mathbf{1 . 9 1}$ & $\mathbf{1 . 3 3}$ & $\mathbf{2 . 7 4}$ & 1.15 & 0.78 & 1.68 & 0.76 & 0.53 & 1
\end{tabular}




\begin{tabular}{|c|c|c|c|c|c|c|c|c|c|c|c|c|c|c|c|c|c|}
\hline \multirow[b]{2}{*}{$\begin{array}{l}\text { People engaged in } \\
\text { sex work }\end{array}$} & \multirow{2}{*}{$\begin{array}{l}\text { aOR } \\
1.00\end{array}$} & \multirow[t]{2}{*}{$95 \% \mathrm{Cl}$} & \multirow{2}{*}{$\begin{array}{l}\text { aOR } \\
0.56\end{array}$} & \multicolumn{2}{|c|}{$95 \% \mathrm{Cl}$} & \multirow{2}{*}{$\begin{array}{l}\text { aOR } \\
0.52\end{array}$} & \multicolumn{2}{|c|}{$95 \% \mathrm{Cl}$} & \multirow{2}{*}{\begin{tabular}{|l|} 
aOR \\
1.78
\end{tabular}} & \multicolumn{2}{|c|}{$95 \% \mathrm{Cl}$} & \multirow{2}{*}{$\begin{array}{l}\text { aOR } \\
1.07\end{array}$} & \multicolumn{2}{|c|}{$95 \% \mathrm{Cl}$} & \multirow{2}{*}{\begin{tabular}{|l|} 
aOR \\
1.18
\end{tabular}} & \multicolumn{2}{|c|}{$95 \% \mathrm{Cl}$} \\
\hline & & & & 0.37 & 0.85 & & 0.35 & 0.78 & & 1.23 & 2.57 & & 0.72 & 1.60 & & 0.81 & 1 \\
\hline $\begin{array}{l}\text { People who } \\
\text { are/have been } \\
\text { incarcerated }\end{array}$ & 1.00 & & 0.86 & 0.57 & 1.29 & 0.72 & 0.48 & 1.06 & 2.20 & 1.53 & 3.16 & 2.41 & 1.64 & 3.56 & 1.92 & 1.33 & 2 \\
\hline $\begin{array}{l}\text { People living with } \\
\text { HIV }\end{array}$ & 1.00 & & 0.39 & 0.25 & 0.59 & 0.45 & 0.30 & 0.68 & 0.99 & 0.68 & 1.45 & 0.61 & 0.40 & 0.91 & 0.58 & 0.40 & 0 \\
\hline $\begin{array}{l}\text { People who use } \\
\text { drugs }\end{array}$ & 1.00 & & 0.57 & 0.38 & 0.86 & 0.37 & 0.25 & 0.55 & 1.13 & 0.78 & 1.64 & 1.53 & 1.03 & 2.28 & 1.45 & 1.00 & 2 \\
\hline $\begin{array}{l}\text { Seniors and Older } \\
\text { Adults }\end{array}$ & 1.00 & & 0.40 & 0.27 & 0.62 & 0.36 & 0.24 & 0.54 & 0.35 & 0.24 & 0.50 & 0.61 & 0.41 & 0.92 & 0.44 & 0.30 & 0 \\
\hline $\begin{array}{l}\text { Transgender } \\
\text { people }\end{array}$ & 1.00 & & 0.55 & 0.37 & 0.84 & 0.48 & 0.32 & 0.73 & 0.98 & 0.68 & 1.43 & 1.11 & 0.75 & 1.66 & 0.70 & 0.48 & 1 \\
\hline $\begin{array}{l}\text { Youth and Young } \\
\text { Adults }\end{array}$ & 1.00 & & 0.55 & 0.35 & 0.85 & 0.31 & 0.20 & 0.48 & 0.79 & 0.54 & 1.15 & 0.98 & 0.64 & 1.49 & 0.93 & 0.63 & 1 \\
\hline
\end{tabular}

\section{Discussion}

\section{Primary Findings}

We conducted a discrete choice experiment asking participants to select between competing public health programs and aimed to identify program characteristics associated with the selected programs. Supporting previous findings and current practice in cost-benefit analyses, ${ }^{22,23,31}$ the results of our discrete choice experiment showed that participants were more likely to select interventions that contributed to greater gains in life expectancy. ${ }^{24,26,32}$ We also observed that participants were more likely to select programs focused on treatment as opposed to prevention. The existing literature on this issue has been mixed, with data from the Swiss Household Panel Survey suggesting that the majority of citizens support preventative interventions over treatment-based ones $^{33}$ while other discrete choice experiments have shown a preference for treatments of more severe illness over less severe illness. ${ }^{34}$ Our findings support the latter, showing that participants in Canada prioritize treatment over prevention - a noteworthy finding given that Canada has a public medicare system that itself focuses on treatment (albeit without universal prescription drug coverage) over prevention. These differences could be due to geographic and population differences or some unmeasured traits.

Extending the existing literature, our study also examined the role of specific health conditions and target populations in shaping funding preferences. In doing so, we found that participants were less likely to select interventions targeting stigmatized health conditions (e.g., HIV and STIs) and more likely to select interventions targeting relatively more common chronic health conditions, such as heart disease and diabetes. We argue that stigma against infectious diseases, mental health problems, and substance use likely underlie these preferences. This conforms with other research that highlight the role of stigma in shaping attitudes towards specific health conditions. ${ }^{35-37}$ Similarly, participants were less likely to select interventions tailored to specific sub-populations, favouring instead those that were tailored to the general population. These findings align with previous research by Skedgel, Wailoo, and Akehurst (2015), which showed that participants generally support interventions that maximize life expectancy gains, but that they are willing to prioritize smaller gains to preferred groups over larger gains to less preferred groups. ${ }^{24}$ These findings therefore highlight potential mechanisms in which marginalized groups are disadvantaged by public opinion. Namely, the public's failure to recognize the needs of key populations who face disproportionate disease burden as a result of syndemic factors. Likewise, Norman et al. (2013) showed that participants tend to favour programs for individuals like themselves, which given our sample was selected from the general populations this would contribute to the less support for marginalized individuals. ${ }^{38}$ Optimistically, the present study highlights several notable exceptions to this general pattern: Participants were more likely to select interventions tailored to populations widely known or believed to be affected by specific health conditions. For example, interventions addressing HIV and other STIs were more likely to be selected when they were targeted to gbMSM, migrants and refugees, people engaged in sex work, or people who are or have been incarcerated but were less likely to be selected when tailored to seniors and older adults. This finding aligns with research by Purtle (2020) showing that the public generally believes that evidence should have "a lot of influence" on health policy decisions. ${ }^{39}$ Likewise, these findings conform to work by Shmueli et al. (2017) and Olsen \& Richardson (2013) which demonstrate the public's support for equity and equality in healthcare priorities. ${ }^{40,41}$ Importantly, we note that participants support for these programs may arise from both accurate (i.e., awareness of epidemiological burden) or inaccurate (i.e., assumptions about the "riskiness" of marginalized groups) perceptions of the need for care within these populations. Certainly, gbMSM and other key populations do experience a disproportionate burden of disease from HIV and other STIs, but these burdens are experienced regardless of whether they are recognized by the public. For example, substance use interventions targeted to Indigenous people were not more likely to be selected in our study, despite the fact that tailored and targeted programs are needed to support Indigenous people who use drugs. ${ }^{42-44}$ Likewise, tailored and targeted interventions addressing mental health and substance use are needed for gbMSM, even though this need may not be as widely recognized as their need for interventions addressing HIV and other STIs. ${ }^{45}$ These findings suggest that the public may not be equally and sufficiently knowledgeable about the full range of inequities facing key populations and that if they were, they might be more supportive of tailored and targeted interventions for high-need populations. 


\title{
Limitations
}

The present study has limitations. First, this study leveraged data from a web-based survey advertised on social media platforms resulting in the underrepresentation of several key populations. While iterative proportional fitting was used to address this bias, it is impossible to know the extent to which hidden factors may have influenced recruitment of participants. Second, our study aimed to elicit preferences indirectly using a discrete choice experiment. It is not clear the extent to which this method is vulnerable to favourable reporting. Nor is it clear whether participants would report these biases if directly assessed. This raises questions about the relevance of discrete choices in the public sphere, given that participants' revealed or expressed opinions may or may not have real bearing on their support or expressed dissatisfaction with a potential public health program. Third, the present study does not allow us to understand how and why participants made the choices they did. Additional qualitative research or experimental quantitative methods are needed to explore the ways in which individuals think about these choices. For example, studies could examine how different prompts and primers might influence these decisions. This work is important to confirm our argument that people's preferences for population-specific programming generally arises from the population's awareness or perceptions of needs for these marginalized groups. We acknowledge, however, that stigma and other factors could also play a role in shaping preferences. Further work is needed to assess the relative contributions of these various factors.

\section{Conclusion}

The findings of the present study suggest that the public generally underestimates the need for tailored and targeted public health programming and may undervalue the benefits of preventive public health efforts relative to treatment-based programs. These results highlight the potential utility of educating the public about health inequities experienced by key populations. We found that while participants generally favored interventions designed for the general public, they were willing to support tailored and targeted interventions in select cases: likely those that conformed with their perceptions of need within key populations. Of course, caution should be taken to ensure that health disparities are not inappropriately attributed to the identities of marginalized and oppressed individuals, but rather the broader social and structural determinants that give rise to inequities.

\author{
Abbreviations \\ $\mathrm{aOR}=$ Adjusted Odds Ratio \\ $\mathrm{Cl}=$ Confidence Interval \\ gbMSM = Gay, Bisexual and Other Men Who Have Sex with Men \\ HIV = Human Immunodeficiency Virus \\ STI = Sexually Transmitted Infection
}

\section{Declarations}

\section{Ethics approval and consent to participate}

Ethics review of our study protocol was conducted by the Research Ethics Board at the University of Victoria (\#H21-00244). All methods were carried out in accordance with relevant guidelines. All participants provided informed consent prior to participation in the study.

\section{Consent for publication}

Not applicable

\section{Availability of data and materials}

The datasets used and/or analysed during the current study are available from the corresponding author on reasonable request.

\section{Competing interests}

The authors declare that they have no competing interests

\section{Funding}

This work was supported (in whole or in part) by an Innovation Research Grant from CANFAR, the Canadian Foundation for AIDS Research. KGC, DMM, and NJL are supported by Scholar Awards from the Michael Smith Foundation for Health Research (\#1547, \#16863).

\section{Authors' contributions}


With the assistance of all authors, Dr. Card conceptualized the design of this study, collected the data, conducted the analyses, and wrote the manuscript. All authors provided feedback and revisions to the paper.

\section{Acknowledgements}

The authors would like to acknowledge the participants who completed the survey and Dr. Anita Lal for her support in providing example discrete choice experiments.

\section{References}

1. Kluge E-HW. Resource Allocation in Healthcare: Implications of Models of Medicine as a Profession. Medscape Gen Med. 2007 Mar 21;9(1):57.

2. Porter TM. The Rise of Statistical Thinking, 1820-1900. Reprint edition. Princeton, NJ: Princeton University Press; 1988.348 p.

3. Berestova AV, Orlov SA, Gorenkov RV, Starostin VP. Ethics in medical decision making: an intercultural outlook. Utopía Prax Latinoam. 2019 Dec 8;24:14451.

4. Board on Population Health and Public Health Practice, Institute of Medicine. Culture as a Social Determinant of Health [Internet]. Leveraging Culture to Address Health Inequalities: Examples from Native Communities: Workshop Summary. National Academies Press (US); 2013 [cited 2021 Aug 6]. Available from: https://www.ncbi.nlm.nih.gov/books/NBK201298/

5. Conrad P, Barker KK. The Social Construction of IIIness: Key Insights and Policy Implications. J Health Soc Behav. 2010 Mar 1;51(1_suppl):S67-79.

6. Gregory R, Slovic P, Flynn J. Risk perceptions, stigma, and health policy. Health Place. 1996 Dec 1;2(4):213-20.

7. Europe WHORO for, Policies EO on HS and, Marchildon GP, Allin S, Merkur S. Canada: Health system review. Health Syst Transit [Internet]. 2020 [cited 2021 Aug 7];22(3). Available from: https://apps.who.int/iris/handle/10665/336311

8. Banuri S, Dercon S, Gauri V. Biased Policy Professionals. World Bank Econ Rev. 2019 Jun 1;33(2):310-27.

9. Fassin D. Public health as culture. The social construction of the childhood lead poisoning epidemic in France. Br Med Bull. 2004 Jun 1;69(1):167-77.

10. FitzGerald C, Hurst S. Implicit bias in healthcare professionals: a systematic review. BMC Med Ethics. 2017 Mar 1;18:19.

11. Daniels N. Resource Allocation and Priority Setting. In: H. Barrett D, W. Ortmann L, Dawson A, Saenz C, Reis A, Bolan G, editors. Public Health Ethics: Cases Spanning the Globe [Internet]. Cham (CH): Springer; 2016 [cited 2021 Aug 6]. Available from: http://www.ncbi.nlm.nih.gov/books/NBK435786/

12. McCaughey D, Bruning NS. Rationality versus reality: the challenges of evidence-based decision making for health policy makers. Implement Sci. 2010 May 26;5(1):39.

13. Baum NM, DesRoches C, Campbell EG, Goold SD. Resource Allocation in Public Health Practice: A National Survey of Local Public Health Officials. J Public Health Manag Pract. 2011 Jun;17(3):265-74.

14. Seixas BV, Regier DA, Bryan S, Mitton C. Describing practices of priority setting and resource allocation in publicly funded health care systems of highincome countries. BMC Health Serv Res. 2021 Jan 27;21(1):90.

15. Wrigley A, Dawson A. Vulnerability and Marginalized Populations. In: H. Barrett D, W. Ortmann L, Dawson A, Saenz C, Reis A, Bolan G, editors. Public Health Ethics: Cases Spanning the Globe [Internet]. Cham (CH): Springer; 2016 [cited 2021 Aug 7]. Available from:

http://www.ncbi.nlm.nih.gov/books/NBK435787/

16. McKay C, Reed M. How personalized should we be? A systematic review of tailored \& targeted health communication interventions to improve adherence. Value Health. 2013 May 1;16(3):A46.

17. Peterson A, Charles V, Yeung D, Coyle K. The Health Equity Framework: A Science- and Justice-Based Model for Public Health Researchers and Practitioners. Health Promot Pract. 2020 Aug 19;1524839920950730.

18. Eslava-Schmalbach J, Garzón-Orjuela N, Elias V, Reveiz L, Tran N, Langlois EV. Conceptual framework of equity-focused implementation research for health programs (EquIR). Int J Equity Health. 2019 May 31;18(1):80.

19. Pedrana L, Pamponet M, Walker R, Costa F, Rasella D. Scoping review: national monitoring frameworks for social determinants of health and health equity. Glob Health Action. 2016;9:28831.

20. Jones CM, Clavier C, Potvin L. Adapting public policy theory for public health research: A framework to understand the development of national policies on global health. Soc Sci Med 1982. 2017 Mar;177:69-77.

21. Christian J. When Does Public Opinion Matter? J Sociol Soc Welf [Internet]. 2008 Mar 1;35(1). Available from: https://scholarworks.wmich.edu/jssw/vol35/iss1/7

22. Mehrez A, Gafni A. Preference based outcome measures for economic evaluation of drug interventions: quality adjusted life years (QALYs) versus healthy years equivalents (HYEs). PharmacoEconomics. 1992 May;1(5):338-45.

23. Dolan P, Shaw R, Tsuchiya A, Williams A. QALY maximisation and people's preferences: a methodological review of the literature. Health Econ. 2005 Feb;14(2):197-208.

24. Skedgel C, Wailoo A, Akehurst R. Societal preferences for distributive justice in the allocation of health care resources: a latent class discrete choice experiment. Med Decis Mak Int J Soc Med Decis Mak. 2015 Jan;35(1):94-105.

25. Whitty JA, Lancsar E, Rixon K, Golenko X, Ratcliffe J. A systematic review of stated preference studies reporting public preferences for healthcare priority setting. The Patient. 2014;7(4):365-86.

Page 9/12 
26. Rowen D, Brazier J, Mukuria C, Keetharuth A, Risa Hole A, Tsuchiya A, et al. Eliciting Societal Preferences for Weighting QALYs for Burden of Illness and End of Life. Med Decis Mak Int J Soc Med Decis Mak. 2016 Feb;36(2):210-22.

27. Austen I. 'Horrible History': Mass Grave of Indigenous Children Reported in Canada. The New York Times [Internet]. 2021 May 28 [cited 2021 Aug 9]; Available from: https://www.nytimes.com/2021/05/28/world/canada/kamloops-mass-grave-residential-schools.html

28. Canada PHA of. COVID-19 daily epidemiology update [Internet]. aem. 2020 [cited 2021 Aug 9]. Available from: https://health-infobase.canada.ca/covid19/epidemiological-summary-covid-19-cases.html

29. Gruzd A, Mai P. The State of Social Media in Canada 2020 [Internet]. Scholars Portal Dataverse; 2020 [cited 2021 Aug 8]. Available from: https://dataverse.scholarsportal.info/dataset.xhtml?persistentld=doi:10.5683/SP2/XIW8EW

30. CBC News Canada Poll Tracker [Internet]. CBC News. 2021 [cited 2021 Aug 8]. Available from: https://newsinteractives.cbc.ca/elections/polltracker/canada/

31. Whitehead SJ, Ali S. Health outcomes in economic evaluation: the QALY and utilities. Br Med Bull. 2010 Dec 1;96(1):5-21.

32. Gu Y, Lancsar E, Ghijben P, Butler JRG, Donaldson C. Attributes and weights in health care priority setting: A systematic review of what counts and to what extent. Soc Sci Med 1982. 2015 Dec;146:41-52.

33. Popic T, Schneider SM, Asensio M. Public Opinion on Healthcare in the Context of Economic Crisis: Evidence from Portugal. J Soc Policy. 2019 Oct;48(4):741-64.

34. Luyten J, Kessels R, Goos P, Beutels P. Public Preferences for Prioritizing Preventive and Curative Health Care Interventions: A Discrete Choice Experiment. Value Health. 2015 Mar 1;18(2):224-33.

35. Schulte A. Consensus versus Disagreement in Disease-Related Stigma: A Comparison of Reactions to Aids and Cancer Patients. Sociol Perspect. 2002 Mar 1;45(1):81-104.

36. Sartorius N. Stigmatized Illnesses and Health Care. Croat Med J. 2007 Jun;48(3):396-7.

37. Ablon J. Stigmatized health conditions. Soc Sci Med [B]. 1981 Jan 1;15(1):5-9.

38. Norman R, Hall J, Street D, Viney R. Efficiency and equity: a stated preference approach. Health Econ. 2013 May;22(5):568-81.

39. Purtle J. Public opinion about evidence-informed health policy development in U.S. Congress. Transl Behav Med. 2020 Dec 31;10(6):1549-53.

40. Shmueli A, Golan O, Paolucci F, Mentzakis E. Efficiency and equity considerations in the preferences of health policy-makers in Israel. Isr J Health Policy Res. 2017;6:18.

41. Olsen JA, Richardson J. Preferences for the normative basis of health care priority setting: some evidence from two countries. Health Econ. 2013 Apr;22(4):480-5.

42. Urbanoski KA. Need for equity in treatment of substance use among Indigenous people in Canada. CMAJ Can Med Assoc J. 2017 Nov 6;189(44):E13501.

43. Rowan M, Poole N, Shea B, Gone JP, Mykota D, Farag M, et al. Cultural interventions to treat addictions in Indigenous populations: findings from a scoping study. Subst Abuse Treat Prev Policy. 2014 Sep 1;9:34.

44. Antonio MCK, Chung-Do JJ. Systematic review of interventions focusing on Indigenous adolescent mental health and substance use. Am Indian Alsk Native Ment Health Res Online. 2015;22(3):36-56.

45. Remien RH, Stirratt MJ, Nguyen N, Robbins RN, Pala AN, Mellins CA. Mental health and HIV/AIDS: the need for an integrated response. AIDS. 2019 Jul 15;33(9):1411-20.

\section{Figures}




\section{Randomly Assigned Values}

1. To prevent cancer

2. To prevent diabetes

3. To prevent drug overdoses

4. To prevent heart disease

5. To prevent mental health problems

6. To prevent sexually transmitted infections, including HIV

7. To treat cancer

8. To treat diabetes

9. To treat heart disease

10. To treat mental health problems

11. To treat sexually transmitted infections, including HIV

12. To treat substance use and addiction
1. 1 year
2. 2 years
3. 3 years
4. 4 years
5. 5 years
6. 6 years
7. 7 years
8. 8 years
9. 9 years
10. 10 years

1. General Population,

2. Gay, bisexual, and other men who have sex with men

3. People who use drugs

4. Indigenous people

5. African, Caribbean, and Black people

6. People engaged in sex work

7. Migrants and refugees

8. People living in or recently released from correctional facilities

9. Transgender people

10. People living with HIV

11. Youth and Young Adults

12. Seniors and Older Adults
The questions in this section ask you to participate in a series of 8 exercises that will help us better understand your decision making process.

In each exercise, you should imagine that you are a decision maker working for the government and you are asked to decide which of two programs should be funded.

Each program will impact 1000 people, but the two programs are designed for different populations and have different impacts on life expectancy. Unfortunately, only one of the two programs can be funded in these hypothetical scenarios. We recognize that these scenarios are hypothetical and are not necessarily intended to represent real world programs.

After you complete the 8 exercises, you will be asked about how easy or difficult the decision making was for you.

Exercise 1 of 8: Based on the information provided below about each program, which would you choose to be funded?

\begin{tabular}{|l|l|l|}
\hline & Program 1 & Program 2 \\
\hline $\begin{array}{l}\text { Program } \\
\text { Purpose... }\end{array}$ & & \\
\hline $\begin{array}{l}\text { Expected } \\
\text { Increase in life } \\
\text { expectancy... }\end{array}$ & & \\
\hline $\begin{array}{l}\text { Program } \\
\text { Designed for... }\end{array}$ & & \\
\hline
\end{tabular}

Program 1

Program 2

Figure 1

Design of Discreet Choice Experiment 
$100 \%$

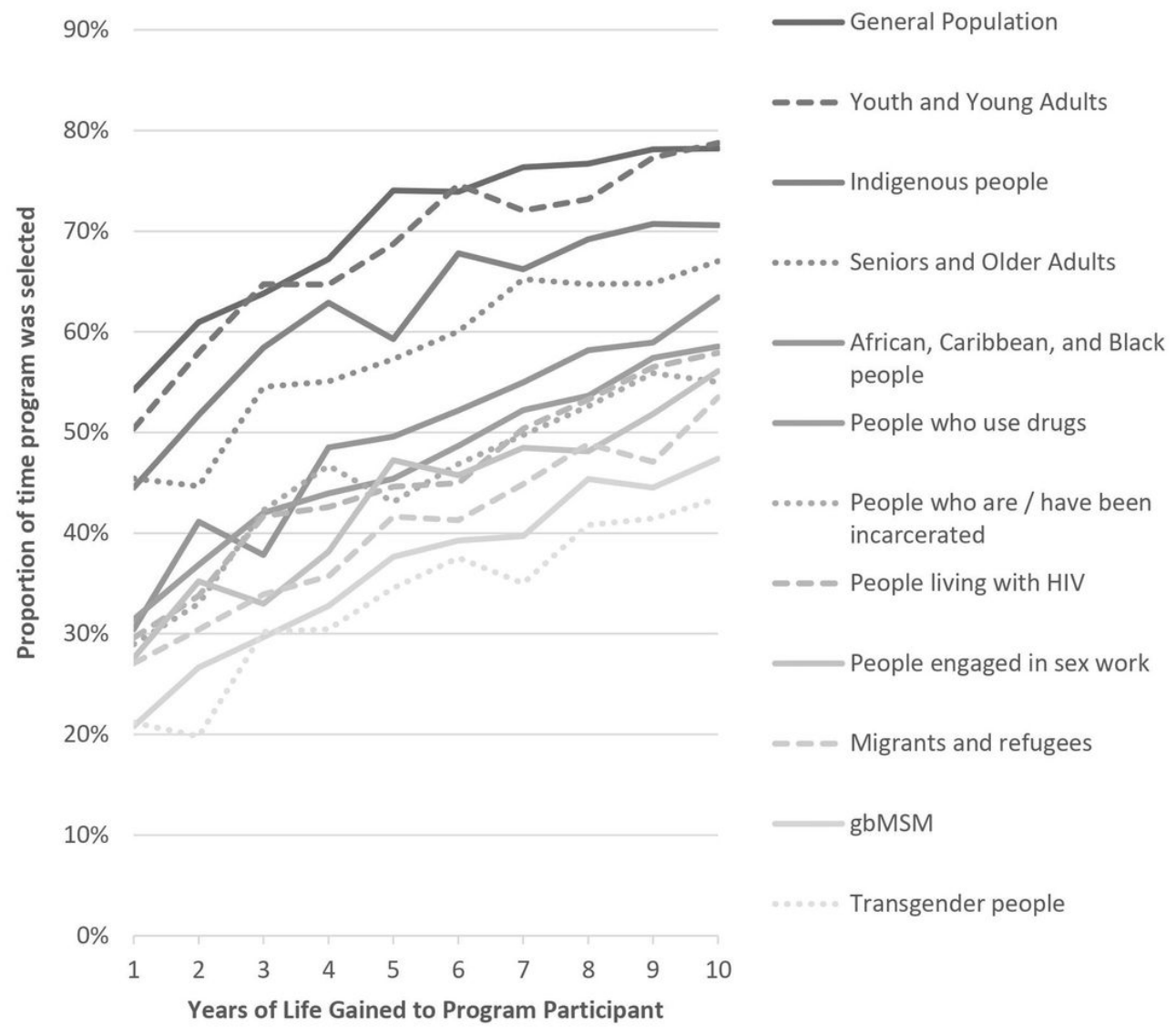

Figure 2

Proportion of time a program serving each client group was selected, by years of life gained to program participant 